\title{
Influence of quantum and thermal noise on spin-torque-driven magnetization switching
}

\author{
Yong Wang, Yan Zhou, and Fu-Chun Zhang \\ Department of Physics, The University of Hong Kong, Hong Kong, China
}

(Received 27 March 2013; accepted 20 June 2013; published online 8 July 2013)

\begin{abstract}
We apply a recently developed quantum theory of spin torque to study the effect of the quantum noise on the magnetization switching. The quantum noise induces considerable fluctuation of the switching time at zero temperature. By including the thermal noise, the temperature dependence of the expectation value and standard deviation of the switching time are obtained, and the results are fitted to an effective first passage model. Our theoretical predictions can be examined in single-shot experiments of spin-torque-driven switching. (C) 2013 AIP Publishing LLC. [http://dx.doi.org/10.1063/1.4813320]
\end{abstract}

In recent years, spin-transfer torque magnetoresistive random-access memory (STT-MRAM) has attracted extensive research interests because of its prospective technological applications. ${ }^{1}$ The basic principle of STT-MRAM is the magnetization switching driven by spin-polarized current in submicro-sized magnetic structures. ${ }^{2,3}$ Although the magnetization dynamics is described well by the Landau-LifshitzGilbert (LLG) equation for many cases, ${ }^{4}$ the performance of the devices is affected or even dominated by the magnetization noises. ${ }^{5-10}$ For example, in the regime of thermally activated magnetization switching, thermal noise plays the dominant role in switching and STT significantly modifies the effective temperature of the devices. ${ }^{11,12}$ While in the dynamical regime, it has been demonstrated that the stochastic trajectories of the STT-driven magnetization switching are induced by the magnetization fluctuations in single-shot measurements. ${ }^{13}$ Therefore, understanding the effect of noise on magnetization switching is desirable for the development of STT-MRAM devices. In addition to the intrinsic quantum noise of the magnetization, there are three sources of noises during spin transfer process: thermal noise from the surrounding environment at finite temperature $;^{5}$ magnetization noise transferred from the current; ${ }^{14,15}$ quantum noise generated from the interaction between current and magnetization. ${ }^{16,17}$ In this letter, we study the effects of these noises on the magnetization switching by the recently developed quantum theory of STT. ${ }^{16,17}$

The device structure is shown in Fig. 1. Between two metallic contacts L1 and L2, the pinned (or fixed) layer M1 and the free ferromagnet (FM) layer M2 are separated by a non-magnetic spacer layer (SL). For simplicity, we assume that M2 is a single-domain magnet. When the electric current passes through M1 under an external bias voltage, it will become spin-polarized and the spin-polarized current will exert spin transfer torque on M2. If the current is larger than a critical value, the magnetization of M2 will be switched to the opposite direction of its original state. In order to take into account the quantum noise during the spin transfer, we apply the method developed in Ref. 16 to study the STTdriven switching dynamics of M2. In this quantum picture, the magnet of M2 is described by the macrospin coherent state $|J, \Theta, \Phi\rangle$, and the current acting on M2 is modelled as a sequence of spin-polarized electrons injected one by one with a fixed time interval $\tau .{ }^{16}$ The motion of the state $|J, \Theta, \Phi\rangle$ is then given by the stochastic Schrödinger equation. ${ }^{16}$ To simulate the Landau-Lifshitz dynamics of the magnet M2, the effective magnetic field and Gilbert damping term are included in the equation for $|J, \Theta, \Phi\rangle$.

In the simulations, the geometry and materials parameters for the magnet $\mathrm{M} 2$ include the saturation magnetization $M$, the cross-section area $A$, the thickness $d$, the Gilbert damping coefficient $\alpha$, and the spin-dependent potential barriers $V_{ \pm}$. Besides, we define the direction of electric current along the $x$ axis, and the electron spin polarization along $z$ axis, as shown in Fig. 1. The demagnetization field $H_{d} m_{x} \hat{e}_{x}$ is along $x$-axis, and the uniaxial anisotropy field $H_{k} m_{z} \hat{e}_{z}$ is along $z$-axis, where $m_{x}$ and $m_{z}$ are the $\mathrm{x}$ - and $\mathrm{z}$-components of the unit vector $\hat{\mathbf{m}}$ of $\mathrm{M} 2$, respectively. ${ }^{18}$ The values of these parameters are listed in Table I. The injected spinpolarized current is characterized by its magnitude $I$ and the spin-polarization ratio $p$. To calculate the scattering matrix, ${ }^{16}$ the electron velocity is set as $v_{g}=3.5 \times 10^{7} \mathrm{~cm} / \mathrm{s}$ for all the electrons.

The trajectories of M2 driven by STT at zero temperature are shown in Fig. 2(a). Here, the initial position of the magnet is $\Theta=3.0$ and $\Phi=\pi / 2$. The current is $I=0.9 \mathrm{~mA}$, and the spin polarization $p=1.0$. Unlike the classical theory, the spin torque here is not given a priori but will show up after the ensemble average of the quantum trajectories generated in the simulations, which also includes the quantum noise. ${ }^{16}$ Nevertheless, the spin torque is known from an alternative approach of the quantum theory ${ }^{17}$ as $\mathcal{A}(\hat{\mathbf{m}} \times \mathbf{S}) \times \hat{\mathbf{m}}+\mathcal{B} \hat{\mathbf{m}} \times \mathbf{S}$, and the quantum noise correlator is ${ }^{17} \frac{\mathcal{A}}{2 J+1}(1-\hat{\mathbf{m}} \cdot \mathbf{S})$. The coefficients $\mathcal{A}$ and $\mathcal{B}$ are proportional to the current $I ;{ }^{17}$ while the electron spin-polarized vector is $\mathbf{S}=(0,0, p)$ for this case. Due to the strong demagnetization field along $x$-direction, the motion of M2 is mostly confined in the $y-z$ plane. The magnet will initially oscillate around the unstable fixed point $\Theta=\pi$, then it switches to the region $\Theta<\pi / 2$ and approaches the stable fixed point $\Theta=0$ in a spiral fashion. Due to the quantum noise during the spin transfer process, ${ }^{16,17}$ the magnetization trajectory becomes stochastic even at zero temperature. Five simulated trajectories of the $\mathrm{z}$-component of the normalized 

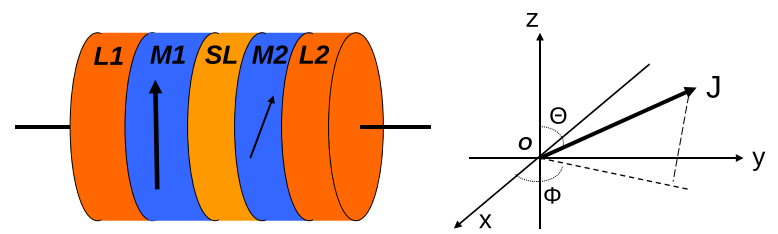

FIG. 1. (Left) Schematic diagram of a spin-transfer torque device. L1/L2: metallic contacts; M1: fixed magnetic layer as spin polarizer. M2: free magnetic layer. SL: spacer layer. (Right) The coordinate for a spin coherent state $|J, \Theta, \Phi\rangle$. Here, $\mathrm{x}$-axis is the electron transport direction, and $\mathrm{z}$-axis is the electron spin-polarizaton direction.

magnetization $\mathrm{J}_{z} / J$ are shown in Fig. 2(a). The switching time $t_{s}$, defined as the interval for the magnet moving from the initial position to $\Theta=\pi / 2$, varies from trajectory to trajectory. The similar feature is observed in the single-shot experiments of magnetization switching, ${ }^{13}$ but the randomness here is completely caused by the quantum noise instead of thermal noise. Furthermore, the statistical distribution of switching time for 1000 simulations is shown in the red histogram of Fig. 2(b). The quantum noise causes a wide distribution of the switching time from $2.8 \mathrm{~ns}$ to $4.6 \mathrm{~ns}$ with the average value of $3.7 \mathrm{~ns}$. This effect is large enough to be observed by the single-shot measurements. ${ }^{13}$ For comparison, we also consider a partially polarized current with $\mathrm{I}=1.8 \mathrm{~mA}$ and $\mathrm{p}=0.5$, which gives the same spin torque magnitude but carries additional noise from the current, as revealed by the torque and noise expressions above. The result is shown in the blue histogram of Fig. 2(b). The distribution of the switching time becomes slightly broader and the average switching time is decreased to $3.68 \mathrm{~ns}$. Thus, the fluctuation of the switching time at zero temperature is mainly due to the quantum noise generated in the spin transfer process, and the injected current noise is less important. This is due to the fact that the magnitudes of the noises are at the same order for the two cases.

The effect of thermal noise on the magnetization switching can be included in the simulations by adding a fluctuating magnetic field $\mathbf{h}_{r}$ defined as ${ }^{5,11,12}$

$$
\left\langle h_{r}^{i}(t)\right\rangle=0, \quad \gamma^{2}\left\langle h_{r}^{i}(t) h_{r}^{j}\left(t^{\prime}\right)\right\rangle=2 D_{T} \delta_{i j} \delta\left(t-t^{\prime}\right),
$$

with the thermal noise correlator $D_{T}=\frac{\gamma \alpha k_{B} T}{M A d}$. Here, $i$ and $j$ are Cartesian indices; $\gamma$ is the gyromagnetic ratio; $k_{B}$ is the Boltzmann constant; and $T$ is the temperature. We have performed 5000 simulations for each of the temperatures $T=0 \mathrm{~K}, 0.5 \mathrm{~K}, 1 \mathrm{~K}, 5 \mathrm{~K}, 10 \mathrm{~K}, 20 \mathrm{~K}, 30 \mathrm{~K}, 40 \mathrm{~K}, 50 \mathrm{~K}, 100 \mathrm{~K}$, $150 \mathrm{~K}, 200 \mathrm{~K}, 250 \mathrm{~K}$, and $300 \mathrm{~K}$ to simulate the statistical distribution of the switching time $t_{s}$. Then the dependence of the mean value $\left\langle t_{s}\right\rangle$ and the standard deviation $\delta t_{s}$ of the switching time on the temperature $T$ are shown in Fig. 3. As the temperature increases, the mean switching time $\left\langle t_{s}\right\rangle$

TABLE I. Parameters for the free layer M2 in the simulations. $M$ : saturation magnetization; $A$ : cross section area; $d$ : film thickness; $H_{d}$ : demagnetization field; $H_{k}$ : easy-axis anisotropy field; $V_{ \pm}$: spin-dependent potential barrier. The units are given in the brackets.

\begin{tabular}{lcccccc}
\hline \hline$M(\mathrm{~A} / \mathrm{m})$ & $A\left(\mathrm{~nm}^{2}\right)$ & $\mathrm{d}(\mathrm{nm})$ & $H_{d}(\mathrm{~T})$ & $H_{k}(\mathrm{~T})$ & $\alpha$ & $V_{ \pm}(\mathrm{eV})$ \\
\hline $1.27 \times 10^{6}$ & 8100 & 1 & 1.6 & 0.03 & 0.02 & $0.7 \pm 0.6$ \\
\hline \hline
\end{tabular}
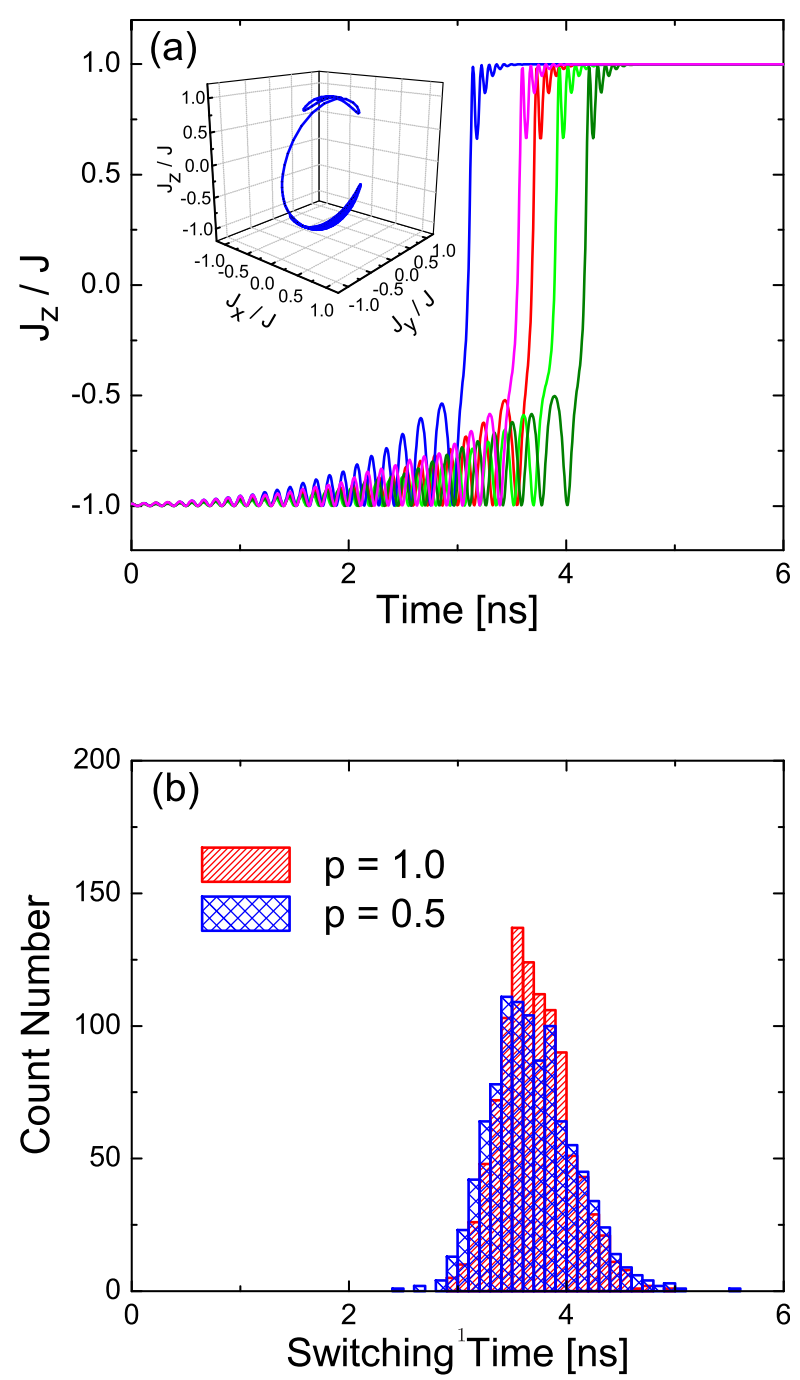

FIG. 2. (a) Five simulated trajectories of the z-component of the normalized magnetization $\mathrm{J}_{z} / J$ at zero temperature. Insert: one typical trajectory of magnetization switching from $\Theta=3.0$ to $\Theta=0$. The applied spin-polarized current is: $\mathrm{I}=0.9 \mathrm{~mA}, \mathrm{p}=1.0$. (b) Histograms of magnetization switching times at zero temperature. Red: $\mathrm{I}=0.9 \mathrm{~mA}, \mathrm{p}=1.0$; Blue: $\mathrm{I}=1.8 \mathrm{~mA}$, $\mathrm{p}=0.5$. For each case, 1000 sample points are used.

decreases monotonically (square symbols), i.e., the thermal noise will assist the magnetization switching for a given STT; the standard deviation of the switching time $\delta t_{s}$, starting from a non-zero value at zero temperature due to the quantum noise, will first increase and then decrease as the temperature increases from $T=0 \mathrm{~K}$ to $T=300 \mathrm{~K}$ (triangles symbols). This is contrary to the intuition that larger thermal noise should cause larger fluctuation of the switching time. This can be understood by considering that the mean switching time $\left\langle t_{s}\right\rangle$ decreases with the temperature. Actually the relative fluctuation of the switching time $\delta t_{s} /\left\langle t_{s}\right\rangle$ indeed increases with the temperature all the way for the temperature range considered here.

We employ a first passage model ${ }^{19}$ to understand the simulation results in Fig. 3. The STT-driven magnetization switching with thermal noise can be regarded as a particle trapped in the interval $\Theta \in[\pi / 2, \pi]$, with the reflecting boundary at $\Theta=\pi$ and absorbing boundary at $\Theta=\pi / 2$. The probability of finding the particle at $\Theta$ is denoted as $\bar{P}(\Theta)$ and satisfies the drift-diffusion equation 


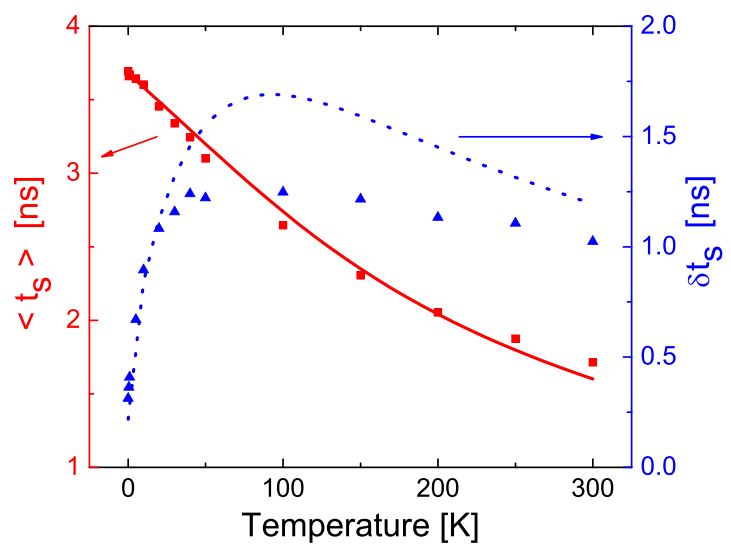

FIG. 3. Dependence of the mean switching time $\left\langle t_{s}\right\rangle$ and standard deviation $\delta t_{s}$ on the temperature $T$. Red squares: $\left\langle t_{s}\right\rangle$ from numerical simulations. Blue triangles: $\delta t_{s}$ from numerical simulations. 5000 simulated trajectories are taken for each temperature. Red solid line: fitting curve for $\left\langle t_{s}\right\rangle$ from Eq. (3). Blue dotted line: fitting curve for $\delta t_{s}$ based on Eqs. (3) and (4).

$$
\frac{\partial}{\partial t} \bar{P}=-\mathcal{T}^{*} \frac{\partial}{\partial \Theta} \bar{P}+\mathcal{D}^{*} \frac{\partial^{2}}{\partial \Theta^{2}} \bar{P}
$$

The drift coefficient $\mathcal{T}^{*}$ is due to the spin torque, while the diffusion coefficient $\mathcal{D}^{*}$ is due to the quantum and thermal noise. For simplicity, $\mathcal{T}^{*}$ and $\mathcal{D}^{*}$ are assumed to be constants, and they are related to the underlying Fokker-Planck equation ${ }^{17}$ for magnetization dynamics through the relations $\mathcal{T}^{*}=\beta_{1} \mathcal{A}$ and $\mathcal{D}^{*}=\beta_{2}\left(\frac{\mathcal{A}}{2 J+1}+D_{T}\right)$. Here, $\frac{\mathcal{A}}{2 J+1}$ is the magnitude of the quantum noise correlator as explained above, ${ }^{17}$ $D_{T}$ is the thermal noise correlator in Eq. (1). With the simulation parameters in Fig. 3, $\mathcal{A}$ is calculated to be $3.5 \mathrm{rad} / \mathrm{ns}$, and the quantum noise is negligible compared with the thermal noise above the critical temperature $1 \mathrm{~K} . \beta_{1}$ and $\beta_{2}$ are two dimensionless parameters characterizing the system, and can be determined by fitting the simulation results in Fig. 3 to the analytical results from Eq. (2). The first two moments of the switching time are given as follows: ${ }^{19}$

$$
\begin{gathered}
\left\langle t_{s}\right\rangle=\frac{\pi^{2}}{4 \mathcal{D}^{*}}\left(\frac{1}{2 P_{e}}-\frac{1-e^{-2 P_{e}}}{4 P_{e}^{2}}\right), \\
\left\langle t_{s}^{2}\right\rangle=\left(\frac{\pi^{2}}{4 \mathcal{D}^{*}}\right)^{2}\left[\frac{1}{4 P_{e}^{2}}-\frac{2-\left(6 P_{e}+1\right) e^{-2 P_{e}}-e^{-4 P_{e}}}{8 P_{e}^{4}}\right] .
\end{gathered}
$$

Here, $P_{e}=\pi \mathcal{T}^{*} / 4 \mathcal{D}^{*}$ is the Péclet number and depends on the temperature through $\mathcal{D}^{*}$. By choosing the fitting parameters $\beta_{1}=0.121, \beta_{2}=350$, the temperature dependence of $\left\langle t_{s}\right\rangle$ and $\delta t_{s}$ are plotted in Fig. 3. The results $\beta_{1} \sim \mathcal{O}\left(10^{-1}\right)$ and $\beta_{2} \sim \mathcal{O}\left(10^{2}\right)$ imply that the oscillations of the magnet before the switching reduce the effective STT but amplify the uncertainty. Such a simplified model gives the excellent fitting curve for $\left\langle t_{s}\right\rangle$ in the whole range of temperatures, but the fitting curve for $\delta t_{s}$ deviates away from the numerical simulations when the temperature is higher than $20 \mathrm{~K}$. It might due to the fact that the oscillations before switching become stronger at higher temperatures and the assumption of constant coefficients $\mathcal{T}^{*}$ and $\mathcal{D}^{*}$ are no longer sufficient. However, the main features of $\delta t_{s}$ are still captured by such a simple treatment. Furthermore, Eqs. (3) and (4) imply that $\delta t_{s} \propto \frac{1}{I} g\left(P_{e}\right)$, where $g\left(P_{e}\right)$ is a universal function of $P_{e}$. Thus, the maximum of $\delta t_{s}$ appears at a fixed value $P_{e}^{*}$, and we can infer that the corresponding temperature is proportional to the current $I$ as long as Eq. (2) holds. We notice that the first passage model has been applied to the thermally activated regime for magnetization switching in a recent work, ${ }^{20}$ while our results here are in the STT-driven dynamical regime and the quantum and thermal noise are perturbative effects.

In conclusion, we have studied the influence of the quantum and thermal noise on the magnetization switching driven by STT. At zero temperature, the stochastic nature of the spin transfer torque on the typical nanomagnet causes significant fluctuation in the switching time of the order of nanoseconds. As the temperature increases and the thermal noise becomes important, the mean switching time decreases monotonically, while the standard deviation of the switching time first increases and then decreases. These results agree reasonably well with a simple first-passage model, and are expected to be observed by the single-shot measurements.

This research was supported by the Hong Kong University Grant Council (AoE/P-04/08).

${ }^{1}$ J. A. Katine and E. E. Fullerton, J. Magn. Magn. Mater. 320, 1217 (2008).

${ }^{2}$ J. C. Slonczewski, J. Magn. Magn. Mater. 159, L1 (1996).

${ }^{3}$ L. Berger, Phys. Rev. B 54, 9353 (1996).

${ }^{4}$ J. Z. Sun, Phys. Rev. B 62, 570 (2000).

${ }^{5}$ W. F. Brown, Phys. Rev. 130, 1677 (1963).

${ }^{6}$ M. Tsoi, A. G. M. Jansen, J. Bass, W.-C. Chiang, M. Seck, V. Tsoi, and P. Wyder, Phys. Rev. Lett. 80, 4281 (1998).

${ }^{7}$ S. Urazhdin, N. O. Birge, W. P. Pratt, and J. Bass, Phys. Rev. Lett. 91, 146803 (2003).

${ }^{8}$ R. H. Koch, J. A. Katine, and J. Z. Sun, Phys. Rev. Lett. 92, 088302 (2004).

${ }^{9}$ D. Bedau, H. Liu, J. Z. Sun, J. A. Katine, E. E. Fullerton, S. Mangin, and A. D. Kent, Appl. Phys. Lett. 97, 262502 (2010).

${ }^{10}$ X. B. Wang, Y. K. Zheng, H. W. Xi, and D. Dimitrov, J. Appl. Phys. 103, 034507 (2008).

${ }^{11}$ Z. Li and S. Zhang, Phys. Rev. B 69, 134416 (2004).

${ }^{12}$ D. M. Apalkov and P. B. Visscher, Phys. Rev. B 72, 180405(R) (2005).

${ }^{13}$ Y. Cui, G. Finocchio, C. Wang, J. A. Katine, R. A. Buhrman, and D. C. Ralph, Phys. Rev. Lett. 104, 097201 (2010).

${ }^{14}$ J. Foros, A. Brataas, Y. Tserkovnyak, and G. E. W. Bauer, Phys. Rev. Lett. 95, 016601 (2005).

${ }^{15}$ A. L. Chudnovskiy, J. Swiebodzinski, and A. Kamenev, Phys. Rev. Lett. 101, 066601 (2008).

${ }^{16}$ Y. Wang and L. J. Sham, Phys. Rev. B 85, 092403 (2012).

${ }^{17}$ Y. Wang and L. J. Sham, Phys. Rev. B 87, 174433 (2013).

${ }^{18}$ Y. Zhou, J. Persson, and J. Akerman, J. Appl. Phys. 101, 09A510 (2007).

${ }^{19}$ S. Redner, A Guide to First-Passage Processes (Cambridge University Press, New York, 2001), p. 54.

${ }^{20}$ D. Pinna, A. Mitra, D. L. Stein, and A. D. Kent, Appl. Phys. Lett. 101, 262401 (2012). 